Variations sur la lettre, le mètre et la mesure :

Shakespeare

\title{
Le mètre et la mesure
}

\section{Henri Suhamy}

\section{(2) OpenEdition}

12 Journals

\section{Édition électronique}

URL : http://journals.openedition.org/shakespeare/963

DOI : 10.4000/shakespeare.963

ISSN : 2271-6424

Éditeur

Société Française Shakespeare

\section{Édition imprimée}

Date de publication : 1 novembre 1996

Pagination : 7-18

\section{Référence électronique}

Henri Suhamy, "Le mètre et la mesure », Actes des congrès de la Société française Shakespeare [En ligne], 14 | 1996, mis en ligne le 01 janvier 2007, consulté le 01 mai 2019. URL : http://

journals.openedition.org/shakespeare/963 ; DOI : 10.4000/shakespeare.963 


\section{Variations sur la lettre,}

le mètre et la mesure

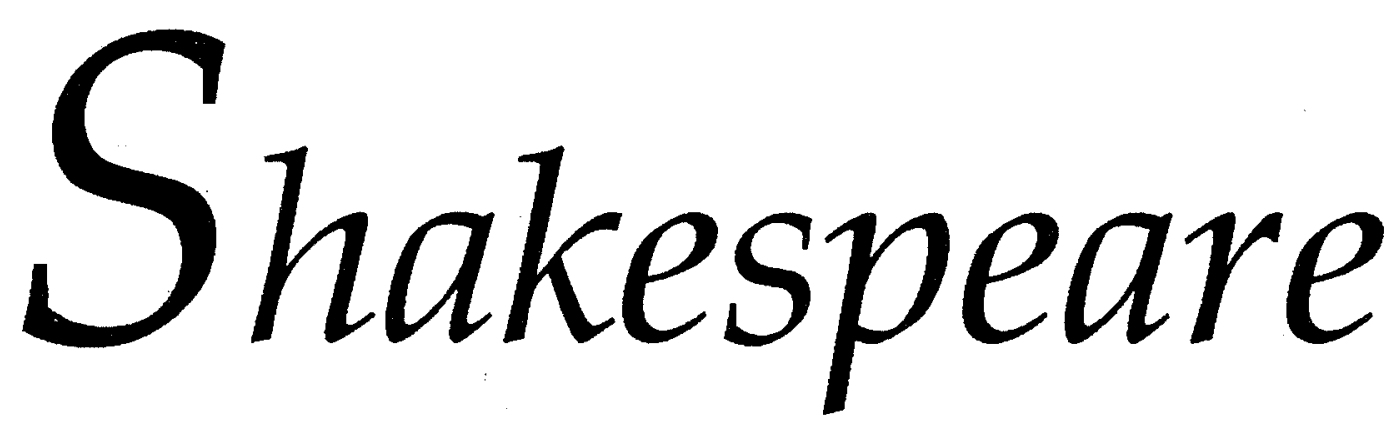

textes présentés par Dominique Goy-Blanquet

Directeur de la publication : Richard Marienstras 


\section{Le mètre et la mesure}

En donnant pour titre à cette communication "Le mètre et la mesure" j'ai conscience de commettre au moins virtuellement une sorte de tautologie, ou de battologie, puisque le grec metron et le latin mensura, d'où proviennent les deux termes, avaient à peu près la même signification, couvrant la notion de mesure. Si l'on remonte encore plus loin, jusqu'au mythique et pourtant bien réel indo-européen dont les philologues de jadis étaient friands, on trouve une origine commune, comme en témoigne l'existence, entre autres formes, du sanscrit matram et du germanique messen. D'après les philologues mentionnés plus haut, la notion de mesure est associée, à l'origine des langues indo-européennes, à l'idée de périodicité, de retour attendu et régulier, ce qui ne manque pas de pertinence en matière de versification. C'est pourquoi les mots qui, dans plusieurs langues, désignent la lune et le mois appartiennent à la même famille. En anglais metre, measure, moon et month sont donc lointainement apparentés.

Cela étant rappelé, les linguistes nous mettent en garde contre le danger de faire de l'étymologie un étalon sémantique. C'est l'usage, particulièrement l'usage actuel et synchronique, qui répond de la signification des mots. Les descendants actuels de deux mots, l'un grec, l'autre latin, qui en leur temps, avaient peut-être la même signification, peuvent très bien s'être spécialisés, voire séparés, dans l'usage que nous en faisons, et cette distinction a certainement son utilité. Si l'usage présente parfois des aspects épineux et irritants, s'il nous impose une tyrannie à la fois vague et contraignante, il nous assure au moins que dans le cas examiné ici il n'existe plus de stricte synonymie entre le mètre et la mesure, même s'il convient de garder en mémoire l'origine commune des deux vocables.

La différence sémantique qui sépare ces deux unités lexicales a un aspect quantitatif. Alors que le mot mètre s'est spécialisé assez étroitement, le mot mesure possède dans l'usage une vaste polysémie. Shakespeare lui-même en fournit la preuve. Le vocable measure, sous ses diverses formes verbales, nominales et adjectivales - il s'agit en l'occurrence de l'adjectif measureless — apparaît 120 fois dans son œuvre, en revêtant des acceptions diverses. Par chance nous pouvons presque toujours le traduire par mesure ou mesurer, sans tomber dans le piège du calque homographique. Cela permet aussi de constater qu'il y a beaucoup de similitudes entre les deux polysémies, celle de l'anglais et celle du français. Cela facilite notre travail, mais sur les autres points il ne faut pas s'attendre à trouver un terrain stable et débroussaillé. La versification anglaise reste un domaine très controversé et rébarbatif.

C'est du mot mètre, et surtout de son homographe anglais metre que viennent les principales difficultés. A cause d'une dérive métonymique qui a commencé dans les années vingt de ce siècle et s'est accentuée dans les années trente, le mot metre 
semble avoir oublié ses liens de parenté avec la notion de mesure. Comme la rupture avec les anciennes dénotations et connotations n'est pas encore complètement consommée, il en résulte une grande confusion lexicale. Il en résulte aussi, tel est du moins le diagnostic sévère que j'ose formuler, une sorte d'atrophie partielle de la sensibilité, une anesthésie, dans la manière qu'ont les gens d'appréhender le phénomène poétique. On pourrait accuser Shakespeare d'avoir donné l'exemple de la dérive métonymique, parce qu'il utilise le mot metre dans un sens vague et général. En fait il commet une synecdoque plutôt qu'un détournement frauduleux ou étourdi. La recension indique que le mot est employé quatre fois seulement, et toujours en relation avec la poésie. Ce détail est intéressant, mais non inattendu, puisque ce mot n'a pas la variété sémantique de son cousin measure et qu'il a un champ d'application beaucoup plus technique et spécialisé, comme indiqué précé-demment. Dans Richard II (II.1.28) le duc d'York parle avec désapprobation des "lascivious metres" qui corrompent la jeunesse et dans la première partie d'Henri $I V$ (III.1.172) c'est Hotspur qui fustige "one of these same metre ballad-mongers". On rencontre l'expression "in metre" dans Measure for Measure (I.2.36) mais je me garderai de tout rapprochement entre cette citation et le titre de la pièce. ${ }^{1}$ On rencontre également le mot metre dans le sonnet 17 , sur lequel je reviendrai. Dans les trois textes cités plus haut on peut avoir l'impression que l'auteur aurait pu aussi bien utiliser le mot verse, mais qu'il a préféré recourir au procédé de la synecdoque, figure de substitution qui consiste à désigner le tout par la partie. Le mètre étant l'un des paramètres du vers, il s'agit d'une partie incluse dans un ensemble. Shakespeare s'appuie sans doute sur la certitude que ses auditeurs comprennent le sens du mot metre, savent qu'il s'agit d'une des composantes de la versification, et reconnaissent instinctivement la projection sémantique qui s'opère d'un signifié à l'autre. Il n'y a sans doute pas de risque de confusion. Tout le monde comprend ce que signifie lascivious metres, même si le recueil ne contient pas d'illustrations.

Mais aujourd'hui, qui comprend le sens technique du mot metre ? A-t-il pour le public actuel la même acception que celle que lui donnait probablement Shakespeare avant d'en étendre le sens à toute la versification ? Probablement pas. Il a été question plus haut de dérive métonymique et de confusion. Voici pourquoi, à travers quelques exemples caractéristiques. Ainsi le livre d'Enid Hamer, The Metres of English Poetry, publié par Methuen en 1930, qui connut un certain succès et fut plusieurs fois réédité, l'article célèbre de C. S. Lewis intitulé simplement "Metre", inclus dans la Review of English Literature, $1960, \mathrm{n}^{\circ} 1$, où il s'en prend violemment à ses prédécesseurs ainsi que, sans le savoir, à ses successeurs, comme Seymour Chatman, auteur d'un livre intitulé A Theory of Meter (graphie américaine), publié par Mouton en 1965, donnent tous au mot metre un sens qui n'a plus aucun lien avec la notion de mesure. Si d'autre part on consulte les différents dictionnaires de terminologie littéraire qui ont été publiés récemment, par exemple celui de Cullen chez Penguin, on constate que contrairement aux définitions anciennes, ces auteurs emploient le mot metre sans se référer à la mesure, même obliquement. Il définissent metre, ou s'y réfèrent implicitement, comme désignant le dessin acoustique produit dans la chaîne du discours par la répartition des syllabes 
accentuées et des syllabes inaccentuées, ou, pour s'exprimer autrement, par leur position les unes par rapport aux autres. Il n'est jamais question, sous la plume de ces auteurs, de la longueur des phrases, de la segmentation du discours en tranches mesurées à l'avance. Cette omission est-elle raisonnable et justifiée en quelque manière ? Je ne le crois pas et j'essaie ici d'expliquer pourquoi.

Dans A Midsummer Night's Dream, pendant la scène 2 de l'acte $\mathrm{I}$, des vers 27 à 34, Bottom improvise ou cite de mémoire un petit poème burlesque et mythologique, qui parodie un passage de l'Hercule furieux de Sénèque :

The raging rocks

And shivering shocks

Shall break the locks

Of prison-gates;

And Phobus' car

Shall shine from far

And make and mar

The foolish fates.

Bien que ce texte ait été imprimé en prose dans le quarto de 1600 ainsi que dans le folio de 1623, les éditeurs ont tous rétabli l'écriture versifiée dans la typographie, ce qui n'a jamais soulevé de protestation. Et pour continuer dans la voie rassurante du consensus, rassurante au vu des controverses qui agitent sempiternellement le petit monde des métriciens, il y a dans ce poème un certain nombre de caractéristiques sur lesquelles tout le monde peut s'accorder. Il est inutile de parler des allitérations et des assonances, car sur leur présence le consensus est presque trop facile. Il faut s'occuper des structures fondamentales du vers. Tout le monde admettra que ce sont des vers ïambiques. On reconnaît le battement, l'alternance ïambique, le mouvement ascendant. Même si au cours d'une discussion quelqu'un insiste pour que l'on prononce shivering en trois syllabes au lieu de shiv'ring, afin d'introduire une modulation ou résolution anapestique, selon la terminologie de George Saintsbury, cela ne représente qu'un accident passager et ne change pas la tendance générale, la cadence de l'ensemble. Mais cela ne suffit pas à décrire la facture de ces vers. Il faut bien aussi prendre les rimes en considération, et admettre que outre leur fonction propre (entre autres choses, créer des similitudes de sons là où il n'y a pas de similitude de sens), elles renforcent l'effet de clôture qui se produit en fin de vers. Et puisque clôture il y a, on peut d'un coup d'œil ou d'un coup d'oreille évaluer, jauger, mesurer la quantité de parole qui précède la rime et la clôture. On constate à ce moment-là, si on ne l'a pas fait avant, que ce sont des vers de quatre syllabes, ou si l'on préfère, de deux pieds. Mais qu'on les appelle tétrasyllabes ou dimètres, on les considérera nécessairement comme des vers courts, par rapport aux normes les plus courantes. La notion de mesure est en train de faire sa réapparition.

Autre exemple : dans Love's Labours Lost (IV.2.101), Nathaniel lit le sonnet que Berowne, ou Biron, a composé pour Rosaline, et qu'il a intercepté : 
If love make me forsworn, how shall I swear to love?

Ah! Never faith could hold, if not to beauty vow'd;

Though to myselfforsworn, to thee I'll faithful prove;

Those thoughts to me were oaks, to thee like osiers bow'd.

Nous pouvons à propos de ce poème comme du précédent tomber d'accord sur un certain nombre de points. Nous avons affaire une fois de plus à des vers ïambiques, même si ce caractère ïambique, c'est-à-dire l'alternance qui va de la brève à la longue ou de la faible à la forte est un peu moins marqué, un peu moins martelé et régulier que dans l'exemple précédent. Mais on peut parler de dominante iambique, qui après une ou deux incartades, impose son mouvement pendulaire, particulièrement sensible dans le quatrième vers : "Those thoughts to me were oaks, to thee like osiers bow'd". On remarque au passage l'omission du pronom that après thoughts, peut-être justement pour obéir à ce que tout le monde continue d'appeler le mètre, non quelquefois sans inconséquence. Autre donnée élémentaire, ce sont des vers rimés, et quand le lecteur arrive à la rime, un peu à bout de souffle, il constate qu'ils ont douze syllabes. Toujours dans un esprit de conciliation œecuménique, considérons comme admissibles les termes d'alexandrins, de dodécasyllabes, de vers de six pieds ou hexamètres, voire de vers de six accents. En tout état de cause il faut admettre aussi que ce sont des vers longs, qui demandent une certaine quantité de souffle, un effort pour tenir la mélodie. Comparés à ceux de Bottom de tels vers produisent un effet différent. Ils traduisent une inspiration différente, et il faut prendre le mot inspiration également au sens physique.

La conclusion provisoire à laquelle je voulais arriver est simplement celleci : la comparaison entre les deux poèmes rappelle que la poésie ïambique peut présenter des visages différents, car la longueur, c'est-à-dire la durée de vers, entraîne un certain type de phrase et d'émission vocale. Si l'on met en avant une expression prétendument savante comme mètre iambique on s'enferme dans une impasse. On se condamne à ne pas percevoir la différence entre les vers courts, les vers longs, et ceux qui n'étant ni courts ni longs correspondent à un débit normal et naturel. Le mot mètre ayant déjà servi à autre chose, en l'occurrence à désigner le dessin accentuel, on ne peut plus le réutiliser. Or, à partir de là, le vocabulaire manque. Il y a dans le domaine de la versification comme partout une partie technique dans laquelle on ne peut pénétrer qu'à l'aide d'une terminologie adéquate. Sans elle on risque de ne pas prendre conscience de certains phénomènes. Les spécialistes qui ont détourné le mot mètre ou son équivalent anglais de leur sens premier et ont oublié leur parenté étymologique avec le concept de mesure n'ont pas proposé de remplaçant. Il convient donc de le récupérer. L'adoption par le Royaume Uni du système métrique devrait faciliter ce retour. Mais alors par quoi remplacer les expressions mètre ïambique, mètre dactylique, etc., si nous décidons de nous en séparer? Elles désignent, comme indiqué plus haut, une alternance, un mouvement pendulaire, une poussée sinusoïdale, un balancement, une pulsion. En anglais, les mots beat ou lilt évoquent assez bien le phénomène. Mais pourquoi ne pas recourir au mot rythme, que tout le monde connaît et utilise abondamment, mais qui malgré sa popularité ou à cause d'elle, mène depuis longtemps la vie errante d'un signifiant 
en quête de signifié? Si l'on reparlait de rythme ïambique, spondaïque ou autres, ce vocable retrouverait un sens intelligible et opératoire, conforme à des usages anciens et respectables, comme en témoignent le dictionnaire de Littré ${ }^{2}$ ainsi que 1'Oxford English Dictionary ${ }^{3}$ Dans le couple indissociable mais non interchangeable que constituent le mètre et le rythme les usagers comprendraient mieux qu'aujourd'hui que le rythme représente une structure purement formelle, non quantifiable, donc non mesurable. En effet ce qui caractérise la pulsation ïambique, trochaïque ou autre est la position respective des syllabes fortes et faibles, non le nombre de leurs occurrences à chaque vers. On se reportera aux deux exemples cités plus haut.

Puisque le mot nombre vient d'apparaître, arrêtons-nous un instant sur lui pour observer, au risque de commettre une lapalissade de plus, qu'il se réfère à une donnée intrinsèquement mesurable. Le nombre, dans la poésie versifiée, a des états de service qui ne méritent pas la négligence ou le mépris. Personne n'a oublié le vers célèbre et autobiographique d'Alexander Pope (Epistle to Dr. Arbuthnot, 128), "I lisp'd in Numbers, for the Numbers came". Pourtant le public ne sait pas toujours que Shakespeare a utilisé la même métonymie une quinzaine de fois. Ainsi quand dans Romeo and Juliet Mercutio fait allusion à Pétrarque, "Now is he for the numbers that Petrarch flowed in", (II.4.42), il se réfère en principe à la poésie italienne, dont les mètres, c'est-à-dire les étalonnages qui fixent la longueur des vers, sont fondés sur l'isosyllabisme, comme en français classique. Dans le domaine de la poésie anglaise, où tout donne lieu à des controverses, certains spécialistes comptent les syllabes, d'autres les pieds, d'autres les accents, il y a aussi ceux qui ne comptent rien du tout et ceux qui comptent sur l'ignorance du public, mais de toute façon il n'est pas inutile de savoir compter, surtout si l'on veut comprendre de quoi parle Mercutio. Il faut bien alors utiliser la notion de nombre. Et ce n'est pas seulement à propos de la poésie italienne que Shakespeare parle de nombres. Il applique ce terme à ses propres vers, notamment quand cela lui donne l'occasion d'un jeu de mots, comme dans le sonnet 17 : "And in fresh numbers number all your graces".

Cet exemple parmi d'autres permet de faire comprendre l'intérêt qu'il y a à réinvestir le mot mètre de sa fonction première, liée à l'idée de mesure. Exemple d'autant plus convaincant que dans ce même sonnet 17 , qui est un poème sur la poésie, on trouve une utilisation du mot metre 4 un peu moins neutre que dans les passages cités précédemment: "The stretched metre of an antique song". Le participe passé stretched, qu'il faut ici prononcer stretchéd, en lui appliquant la notion même qu'il exprime, se réfère manifestement à l'aspect dimensionnel de la versification. Il ne peut pas désigner un élément purement accentuel et pulsionnel, ni une configuration comme celles auxquelles on pense quand on parle de vers ïambiques ou trochaïques. Incidemment on remarque que le mot stretched contient un autre jeu de mots, qui tient du concetto : il se réfère à la notion d'exagération. En vantant la beauté de son ami par des dithyrambes hyperboliques, l'auteur risque d'être accusé plus tard d'avoir tiré un peu trop sur la corde élogieuse, d'avoir étiré le procédé. Mais le fait d'avoir utilisé metre au singulier lui confère une dénotation qui conserve en partie le sens propre. Shakespeare confirme ici, après avoir écrit le mot 
numbers six vers plus haut, qu'il donne à metre un sens quantitatif plutôt que rythmique et formel. Ce terme doit donc, dans l'esprit de Shakespeare, se référer au découpage du discours en ces tranches approximativement isochrones que l'on appelle les vers.

Cela suscite inévitablement la question suivante, que pourtant on n'ose souvent pas poser : à quoi sert ce découpage, quelle est la raison d'être de la régularité qui en résulte, et de sa monotonie ? Cette question a souvent été posée, depuis que la poésie versifiée existe, c'est-à-dire depuis des millénaires, et des réponses ont été fournies. Mais sur les origines et les fins de toutes choses chaque génération pose les mêmes questions et entend ou se donne des réponses différentes. D'ailleurs, en matière de création artistique comme en matière de Création tout court, il n'y a peut-être pas de distinction à établir entre les origines et les finalités. A la question en question, donnons précisément la mesure pour réponse. Le discours versifié peut donc se définir comme une déclamation mesurée, une suite harmo-nieuse de paroles et de silences, chaque vers combinant, en étroite symbiose, une certaine quantité d'air en provenance des poumons, une émission sonore articulée par l'appareil phonique, et une production verbale énoncée de façon à former une unité syntaxique, le tout étant calibré selon les conventions métriques et balisé par la rime, ou à défaut de rime, par une pause rituelle. Il faut à cette liste ajouter la pulsation rythmique, appelée improprement mètre dans certains manuels. Chaque vers constitue un souffle, une mélodie, une phase et une phrase, un moment de notre vie et de notre pensée. Les vers se suivent régulièrement comme les vagues de l'océan, et trois siècles avant que l'auteur français d'un poème en décasyllabes ait interpellé la mer, la mer toujours recommencée, un autre pratiquant du décasyllabe en série utilisa l'image maritime à la fois pour décrire le cours de la vie et former la matrice du poème qu'il était en train d'écrire. Il s'agit du sonnet 60 , transcrit cidessous d'après l'édition de 1609 :

Like as the waues make towards the pibled shore, So do our minuites hasten to their end, Each changing place with that which goes before, In sequent toile all forwards do contend. Natiuity once in the maine of light, Crawles to maturity, wherewith being crown'd, Crooked eclipses gainst his glory fight, And time that gaue, doth now his gift confound. Time doth transfixe the florish set on youth, And delues the paralels in beauties brow, Feedes on the rarities of natures truth, And nothing stands but for his sieth to mow. And yet to times in hope, my verse shall stand Praising thy worth, dispight his cruell hand.

La forme de ce poème est déterminée, calculée, mesurée, le nombre de vers fixé d'avance, le nombre de syllabes également, le rythme accentuel est volontairement 
binaire et la diction aménagée de façon que les phrases composant le discours aient une durée à peu près égale et soient séparées par de courtes plages de silence. Il ne faut pas donner au mots phrases un sens trop strictement grammatical, mais on peut constater que chaque émission vocale possède une certaine unité et cohérence syntaxiques. Le silence en fin de vers, c'est-à-dire ce qu'en langage musical autant que physiologique on appelle la respiration, constitue un élément essentiel du vers, bien que peu de spécialistes en parlent, mais contrairement aux commentateurs universitaires les poètes eux-mêmes connaissent l'importance du silence quand ils parlent de leur art ainsi que, visuellement, celle de l'espace que l'écriture versifiée laisse en blanc sur la page. Rappelons que le mot vers, du latin versus, implique l'idée d'un arrêt en fin de trajectoire et d'un recommencement.

La mesure du décasyllabe, le mot étant utilisé ici au sens où l'on parle d'une mesure de blé, d'une mesure d'huile, mérite une mention spéciale, car c'est celle qué Shakespeare et la poésie anglaise en général ont employée le plus couramment. Nous avons vu que les vers très courts de Bottom produisent un effet de compression parodique tout en favorisant l'énergie pulsative du rythme. A l'inverse les alexandrins de Biron ne se déroulent pas sans quelque alanguissement et demandent au récitant d'emmagasiner une quantité de souffle peut-être inhabituelle. Le décasyllabe, appelé aussi pentamètre, occupe une position privilégiée, qui vient probablement de ce que sa durée d'émission correspond à peu près à celle d'une expiration normale et tranquille. En se forçant à calquer ses phrases et parties de phrases sur les allées et venues régulières de l'air dans les poumons ainsi que sur un nombre de syllabes fixé à l'avance, le poète instaure une harmonie profonde et préétablie entre la pensée et le corps. L'esprit, spiritus, retrouve grâce à la poésie déclamée sa dualité et son unité. Par sa substance, son unité syntaxique, sa courbe intonative et son rythme, chaque vers constitue un air, au sens physique et mélodique.

Ces aperçus théoriques soulèvent des objections bien connues. Les descriptions qui conviennent au sonnet 60 et à ses énoncés aphoristiques ne s'appliquent pas nécessairement à toute la poésie versifiée, ni même à celle de Shakespeare dans son ensemble. L'absence de rimes qui caractérise une grande partie du discours dramatique empêche souvent de percevoir auditivement la fin des vers, surtout quand il y a des enjambements, rejets et contre-rejets. Entre la construction syntaxique et la segmentation métrique il se produit toutes sortes de tiraillements et de débordements. Le rythme lui-même subit des irrégularités, des variations verticales et horizontales, dues à divers facteurs, notamment l'intonation de la phrase. Le décompte des syllabes, parfois difficile à établir avec précision, fait apparaître des syllabes en trop ou en moins par rapport au schéma de base. Je me contenterai ici d'aborder la question des enjambements, parce qu'elle revient à se demander si, dans la pratique, le vers shakespearien observe toujours la mesure, et de quelle façon. Pour éclairer le débat je propose une expérience, en demandant au lecteur ou à la lectrice d'en tirer la leçon. Lire deux fois de suite le célèbre monologue de Hamlet, (III.1.56-88), la première fois en suivant la pente naturelle des phrases, leur logique propre, le sentiment qui les anime, comme si le texte était écrit en prose. La seconde fois, en respectant scrupuleusement la pause en fin de vers, même quand il n'y a aucun signe de ponctuation et que la phrase continue. L'élocution mesurée, vers par 
vers, se calque sur la présentation graphique de la tirade et correspond à une tradition ancienne, fondée sur les considérations esthétiques qui ont fait l'objet des paragraphes précédents. Le texte transcrit ci-dessous vient du quarto de 1604 , complété au vers 83 par un membre de phrase présent dans le texte de 1623 (of vs all). C'est une version intéressante, car la ponctuation semble naturelle, sans doute assez proche de ce que l'auteur a écrit, malgré quelques bizarreries qui ont donné lieu à des discussions :

To be, or not to be, that is the question, Whether tis nobler in the minde to suffer

The slings and arrowes of outragious fortune, Or to take Armes against a sea of troubles, And by opposing, end them, to die to sleepe No more, and by a sleepe to say we end The hart-ake, and the thousand naturall shocks That flesh is heire to; tis a consummation Deuoutly to be wisht to die to sleepe, To sleepe, perchance to dreame, I there's the rub, For in that sleepe of death what dreames may come When we haue shuffled off this mortall coyle Must giue vs pause, there's the respect

That makes calamitie of so long life :

For who would beare the whips and scornes of time, Th'oppressor's wrong, the proude mans contumely, The pangs of despiz'd loue, the lawes delay, The insolence of office, and the spurnes That patient merrit of th'vnworthy takes, When he himself might his quietas make With a bare bodkin; who would fardels beare, To grunt and sweat vnder a wearie life, But that the dread of something after death, The vndiscouer'd country, from whose borne No trauiler returnes, puzzels the will, And makes vs rather beare those ills we haue, Then flie to others that we know not of. Thus conscience dooes make cowards [of vs all], And thus the natiue hiew of resolution Is sickled ore with the pale cast of thought, And enterprises of great pitch and moment, With this regard theyr currents turne awry, And loose the name of action.

La première lecture, réaliste et naturellement discursive, correspond à ce que l'on entend habituellement sur la scène, pas toujours cependant car on observe depuis quelques années une tendance à revenir à une diction plus formalisée. La seconde 
lecture, celle qui justement est formalisée, consiste à respecter et même privilégier le mètre, de façon quasi rituelle et liturgique, en s'efforçant de toujours s'arrêter en fin de vers pour respirer, même quand cela provoque une coupure au milieu d'une phrase, y compris entre un sujet et un verbe ou entre un verbe et un complément d'objet direct. L'enjambement est senti alors non pas comme la victoire passagère de la parole naturelle sur la forme qu'on lui impose arbitrairement, mais au contraire comme la preuve que la forme métrique exerce son empire sur la parole en toutes circonstances, afin de produire une déclamation poétiquement stylisée. Bien entendu mener à bien l'opération exige un certain doigté. La diction dite mesurée apparaîtrat-elle comme simplement artificieuse et conventionnelle, tout juste capable d'introduire une distanciation doublement démodée, ou comme enrichissant le discours d'un ensemble de résonances véritablement poétiques, par l'effet de mélopée qu'elle produit ainsi que par les ruptures et fusions inattendues qui résultent du découpage métrique. Si l'on tombe dans le syndrome de Quince, qui en récitant le prologue de Pyrame et Thisbé désarticule le discours, l'affaire est entendue. Mais il est permis de penser qu'on peut aussi transmettre tout le contenu, y compris émotif, de la tirade, en respectant les linéaments traditionnels de la diction métrique.

Cela étant dit, le choix du monologue de Hamlet n'est peut-être pas le plus spectaculaire, car sans avoir le caractère ouvertement sentencieux du sonnet 60 , il rappelle au moins par son contenu le sonnet 66, "Tired with all these, for restful death I cry", sorte de litanie répétitive, composée d'une série de propositions infinitives ou participiales écrites sur le même modèle, donc aisément superposables. Il y a plus de fluidité dans le monologue du prince, mais en lui imprimant vocalement une structure plus aphoristique que ce que suggère parfois le mouvement des phrases on crée un décalage fructueux. D'ailleurs les deux lectures proposées ne sont pas intégralement différentes, puisque souvent la phrase se coule naturellement dans le moule décasyllabique. La forme métrique ne fait pas toujours violence au langage, elle impose des cadres, des guides, elle stimule la pensée autant qu'elle la contraint.

La question du décalage entre la diction et la syntaxe se complique quand on sait que certains orateurs politiques et autres pratiquent parfois des interruptions inattendues au milieu d'une phrase, entre un sujet et un verbe par exemple, pour réveiller les auditeurs ou susciter une attente. L'effet obtenu appartient à la rhétorique plus qu'à la poétique, mais ces deux activités se ressemblent et utilisent parfois les mêmes procédés. On attend toutefois de la poésie quelque chose de plus mystérieux, de moins directement utilitaire.

Lorsqu'on parcourt l'évolution du style prosodique de Shakespeare et que l'on constate que le discours devient de plus en plus passionné, semble se faire plus parlé que chanté, plus proche du récitatif que de l'aria, les mêmes questions se posent, avec une difficulté accrue, mais rien ne change fondamentalement. On peut parler de différences de degré plus que de nature. Le ton devient plus personnel et introverti, moins déclamatoire, il y a de moins en moins de rimes et de plus en plus d'enjambements, mais pour le récitant ou l'acteur le dilemme reste identique, entre le choix d'une diction naturelle mais prosaïque et celui d'une élocution mesurée, à moins d'être découragé ou dépassé par l'apparence de liberté excessive que présente 
le vers de Shakespeare dans sa dernière phase, et de renoncer à toute tentative de conciliation entre la forme et l'expression. D'autre part parler d'élocution mesurée peut avoir une signification autre que technique et se heurter à un autre type de difficulté. Comment en effet pouvons-nous mentionner le thème de la mesure en présence d'un théâtre et d'un certain nombre de personnages qui se caractérisent notoirement par la démesure ? On peut se méfier d'un idéal de discipline, d'une gymnastique vocale qui auraient pour conséquence de briser les élans, d'édulcorer les passions, de créer une atmosphère froide et compassée, en contradiction avec le contenu du texte. La mesure dans la démesure ne peut certes produire qu'un état paradoxal et instable, difficile à atteindre et à maintenir une fois atteint.

C'est ce dont parlait Wordsworth dans la préface écrite en 1800 pour les Lyrical Ballads, où il explique comment les mots arrangés métriquement procurent du plaisir. Le dessein de la poésie, dit-il, est de produire de l'émotion (excitement), associée à une quantité de plaisir encore plus grande (an overbalance of pleasure). Mais si les mots qui produisent l'émotion sont puissants en eux-mêmes, ou si les sentiments et les images sont associés à une proportion excessive de douleur, l'émotion risque de dépasser les limites convenables. Or la présence simultanée (copresence) de quelque chose de régulier, quelque chose auquel l'esprit a été accoutumé alors qu'il se trouvait dans un état de tranquillité ou de trouble moins intense, ne peut pas manquer de tempérer et d'atténuer la passion en la mêlant intimement à un sentiment plus ordinaire. Plus loin Wordsworth dit que dans les scènes les plus pathétiques de Shakespeare, le pathétisme ne nous emporte jamais au-delà du plaisir, grâce aux effets de surprise agréables, minimes mais continus et réguliers, qui proviennent de l'arrangement métrique. Il ne parle pas des passages en prose, mais cette omission ne peut que confirmer, ou du moins renforcer sa thèse.

En guise de conclusion, voici un dernier texte, à lire à haute voix. Il s'agit d'un passage taillé sur mesure pour un personnage démesuré. Toutefois, sans en minimiser la grandiloquence, il convient de le déclamer en mesure, c'est-à-dire en s'arrêtant toujours à la fin des vers. C'est la fameuse tirade des prisonniers que prononce Hotspur dans la première partie d'Henri IV (I.3.28-68). Avec véhémence, non sans quelque mauvaise foi, il explique devant la cour pourquoi il n'a pas livré ses prisonniers après la bataille de Holmedon, en insérant le portrait burlesque du messager royal. Il est recommandé de respecter certains détails techniques, notamment les diérèses, qui, afin de maintenir les normes métriques, allongent certains mots : ainsi soldier se prononce en trois, impatience en quatre, occupation et accusation en cinq syllabes. Selon les besoins, le mot prisoners se prononce en deux ou en trois syllabes. La désinence du participe perfumed doit être articulée. L'adjectif extreme est accentué sur la première syllabe. Le texte est ici celui du folio de 1623. On remarque l'extrême densité de la ponctuation, en partie effacée dans les éditions courantes. En fait si les signes de ponctuation en fin de vers matérialisent la forme métrique, les nombreuses pauses dans le cours du vers produisent un effet curieusement analogue à celui des enjambements : elles conduisent à une diction hachée, haletante, reflétant un état de nervosité qui contrarie la mélodie tranquille, cathartique du décasyllabe traditionnel. Celle-ci ne s'impose pas ici. La diction la plus respectueuse des traditions ne consiste pas à gommer les accidents, dont l'expres- 
sivité vient précisément de ce qu'ils sont sentis comme des accidents. On notera par exemple l'amusant rejet de "So Cowardly" au vers 62 , mis en valeur par un point que l'édition Arden remplace par une virgule :

My liege, I did deny no Prisoners.

But, I remember when the fight was done, When I was dry with Rage, and extreame Toyle, Breathlesse, and Faint, leaning vpon my Sword, Came there a certaine Lord, neat and trimly drest; Fresh as a Bride-groome, and his Chin new reapt, Shew'd like a stubble Land at Haruest home. He was perfumed like a Milliner, And 'twixt his finger and his Thumbe, he held A Pouncet-box: which euer and anon He gaue his Nose, and took't away againe: Who therewith angry, when it next came there, Tooke it in Snuffe. And still he smil'd and talk'd: And as the Souldiers bare dead bodies by, He call'd them vntaught Knaues, Vnmannerly, To bring a slouenly vnhandsome Coarse Betwitxt the Winde, and his Nobility. With many Holiday and Lady tearme He question'd me: Among the rest, demanded My Prisoners, in your Maiesties behalfe. I then, all smarting, with my wounds being cold, (To be so pestered with a Popingay) Out of my Greefe, and my Impatience, Answer'd (neglectingly) I know not what, He should, or he should not: For he made me mad, To see him shine so briske, and smell so sweet, And talke so like a Waiting-Gentlewoman, Of Guns, \& Drums, and Wounds : God saue the marke; And telling me, the Soueraign'st thing on earth Was Parmacity, for an inward bruise : And that it was great pitty, so it was, That villanous Salt-peter should be digg'd Out of the Bowels of the harmlesse Earth, Which many a good Tall Fellow had destroy'd So Cowardly. And but these vile Gunnes, He would himselfe haue beene a Souldier. This bald, vnioynted Chat of his (my Lord) Made me to answer indirectly (as I said.) And I beseech you, let not this report Come currant for an Accusation, Betwixt my loue, and your high Maiesty. 


\section{LE MÈTRE ET LA MESURE}

Comme toutes les tirades, celle-ci est composée d'air, mais un autre élément s'y ajoute, le feu, car Henry Percy, dit Hotspur, personnage tragique et comique à la fois, incarnation de la démesure, est emporté, quoique en mesure, par son éperon brûlant.

1 Les citations et les références sont tirées des éditions Arden.

2 "Qualité du discours qui, par le moyen de ses syllabes accentuées, vient frapper notre oreille à certains intervalles."

3 "The measured recurrence of arsis and thesis determined by vowel-quantity or stress, or both combined; ..."

4 La graphie miter que l'on trouve dans le texte de 1609 est un peu déconcertante, mais il ne s'agit tout de même pas d'une mitre. 